\title{
Combined supplementation of Lactobacillus fermentum and Pediococcus acidilactici promoted growth performance, alleviated inflammation, and modulated intestinal microbiota in weaned pigs
}

Shilan Wang, Bingqian Yao, Hang Gao, Jianjun Zang*, Shiyu Tao, Shuai Zhang, Shimeng Huang, Beibei He and Junjun Wang ${ }^{*}$ (D)

\begin{abstract}
Background: Probiotics are important for pigs to enhance health and intestinal development, which are potential alternative to antibiotics. Many studies have reported the functions of single bacterial strain as probiotic on the animals. In this study, we evaluated effects of combined probiotics on growth performance, inflammation and intestinal microbiota in weaned pigs. One hundred and eight pigs, weaned at 28 day old $(7.12 \pm 0.08 \mathrm{~kg})$, were randomly divided into the 3 dietary treatments with 6 pens and 6 pigs per pen (half male and half female). The experimental period lasted for 28 days and treatments were as follows: i. Control: basal diet; ii. Antibiotic: the basal diet plus $75 \mathrm{mg} \cdot \mathrm{kg}^{-1}$ chlortetracycline; and iii. Probiotics: basal diet plus $4 \%$ compound probiotics.

Results: Supplementation probiotics improved average daily gain over the entire 28 days $(P<0.01)$ and feed efficiency in the last 14 days $(P<0.05)$ compared with the other two groups. Both probiotics and antibiotic supplementation decreased concentrations of serum pro-inflammatory cytokines interleukin- $6(P<0.05)$ and interferon- $\gamma(P<0.01)$. Probiotics group had greater abundance of Lactobacillus in the caecal digesta and Firmicutes in the colonic digesta, while both probiotics and antibiotic supplementation inhibited Treponema_2 and Anaerovibrio in the caecal digesta. Caecal acetic and propionic acid $(P<0.05)$ of probiotics group were higher than the other two groups, whereas concentrations of colonic lactic acid and propionic acid $(P<0.05)$ of antibiotic group were lower than control and probiotics groups.

Conclusions: These findings suggest that combined supplementation of Lactobacillus fermentum and Pediococcus acidilactici regulate the gut health and improve the host ADG and F/G by decreasing serum pro-inflammatory factors (IL-6, IFN- $\gamma$ ), promoting beneficial bacteria (Lactobacillus in the caecal digesta and Firmicutes in the colonic digesta), enhancing production of short chain fatty acids, and inhibiting pathogens (Treponema_2, Anaerovibrio in the caecal digesta).
\end{abstract}

Keywords: Weaned pigs, Growth performance, Inflammation, Intestinal microbiota, Combined Lactobacillus fermentum and Pediococcus acidilactici

\footnotetext{
*Correspondence: zangjj@cau.edu.cn; wangjj@cau.edu.cn

State Key Laboratory of Animal Nutrition, College of Animal Science and

Technology, China Agricultural University, Beijing 100193, China
}

(c) The Author(s). 2019 Open Access This article is distributed under the terms of the Creative Commons Attribution 4.0 International License (http://creativecommons.org/licenses/by/4.0/), which permits unrestricted use, distribution, and reproduction in any medium, provided you give appropriate credit to the original author(s) and the source, provide a link to the Creative Commons license, and indicate if changes were made. The Creative Commons Public Domain Dedication waiver (http://creativecommons.org/publicdomain/zero/1.0/) applies to the data made available in this article, unless otherwise stated. 


\section{Background}

Weaning is accompanied by changes of intestinal microbiota composition, diarrhea and growth inhibition in weaned pigs $[1,2]$. Supplementation of feed with antibiotics can promote growth and stabilize intestinal microbiota [3], but now antibiotics have to be limited in pig production due to increased resistance of microorganism to antibiotics. The intestinal microbiota plays a pivotal role in benefiting the health of the host animals [4]. Probiotics can stimulate the immune system of piglets, inhibit growth of pathogens, and modulate composition and activity of the original microbiota [4], which are potential alternative to antibiotic, especially for preventive effect.

Recent previous research has reported the functions of lactic acid bacteria strains as probiotics in animals [4]. Some Lactobacillus species can change host intestinal microbiota through producing lactic acid and other microbial compounds, and they may prevent colonization of pathogens via competitive exclusion $[5,6]$. Lactobacillus could use nutrients that the host cannot metabolize, and thus affect physiological functions of animals, such as the general health and growth [7].

Lactobacillus fermentum (L. fermentum) strains improved the anti-oxidative defense system of weanling pigs [8] and prevented intestinal infections caused by enterotoxigenic Escherichia coil [9]. A previous study showed that Lactobacillus fermentum 15007 relieved the weaning stress by decreasing expression of proteins participated in stress response and increasing levels of proteins in relation to protein synthesis, and immune response [10]. Feeding probiotics containing Pediococcus acidilactici ( $P$. acidilactici) could modulate bacterial communities related to intestinal health of weaned piglets $[11,12]$. However, the combined effect of L. fermentum and $P$. acidilactici on weaned pigs has not been investigated extensively. Therefore, we aimed to investigate the combined effects of L. fermentum and P. acidilactici on growth performance, immune function, short chain fatty acid concentrations, and intestinal bacterial communities in weaned pigs in this study.

\section{Results}

\section{Growth performance}

The probiotics group showed significantly high ADG compared to control and antibiotic groups $(P<0.05)$ over the entire 28 days (Table 1 ). During the first 14 days, no significant changes in ADFI and F/G were detected among the treatment groups. During day 15-28, the probiotics group consumed less feed and consequently had lower $F / G$ than the other two groups $(P<$ $0.05)$. A lower $F / G$ trend was observed during the entire 28 days in the probiotics group.

\section{Immune function}

We observed significant effects of probiotics on levels of inflammatory cytokines in the serum (Table 2). Serum levels of IL-6 $(P<0.05)$ and IFN- $\gamma(P<0.01)$ in probiotics and antibiotic groups were decreased than the control group. Feeding probiotics exhibited additional effects in down-regulating interleukin-1 $\beta$ (IL-1 $\beta)(P<0.05)$, whereas feeding antibiotic uniquely

Table 1 Effect of combined probiotics on the growth performance of weaned pigs ${ }^{1}$

\begin{tabular}{|c|c|c|c|c|}
\hline Items & Control & Antibiotic & Probiotics & $P$-value \\
\hline Initial weight (kg) & $7.11 \pm 0.86$ & $7.10 \pm 0.99$ & $7.13 \pm 0.89$ & 0.99 \\
\hline Final weight (kg) & $15.25 \pm 1.01$ & $15.57 \pm 1.34$ & $17.39 \pm 1.36$ & 0.03 \\
\hline \multicolumn{5}{|l|}{ d1-14 } \\
\hline ADG $(g / d)$ & $197.88 \pm 4.59^{b}$ & $199.47 \pm 6.87^{b}$ & $247.38 \pm 23.47^{a}$ & 0.04 \\
\hline ADFI (g/d) & $400.33 \pm 23.93$ & $376.28 \pm 14.84$ & $409.38 \pm 21.53$ & 0.45 \\
\hline$F / G$ & $2.03 \pm 0.14$ & $1.89 \pm 0.09$ & $1.70 \pm 0.17$ & 0.27 \\
\hline \multicolumn{5}{|l|}{ d14-28 } \\
\hline ADG (g/d) & $383.75 \pm 18.45^{b}$ & $405.73 \pm 14.87^{b}$ & $485.75 \pm 23.78^{\mathrm{a}}$ & 0.01 \\
\hline ADFI (g/d) & $739.30 \pm 45.70$ & $779.07 \pm 30.82$ & $876.33 \pm 52.75$ & 0.12 \\
\hline$F / G$ & $1.92 \pm 0.03^{\mathrm{a}}$ & $1.92 \pm 0.03^{\mathrm{a}}$ & $1.80 \pm 0.04^{b}$ & 0.04 \\
\hline \multicolumn{5}{|l|}{ d1-28 } \\
\hline ADG $(g / d)$ & $290.83 \pm 9.47^{b}$ & $302.60 \pm 9.13^{b}$ & $366.58 \pm 12.80^{\mathrm{a}}$ & $<0.01$ \\
\hline ADFI (g/d) & $569.80 \pm 34.61$ & $577.67 \pm 15.42$ & $642.83 \pm 36.90$ & 0.19 \\
\hline$F / G$ & $1.96 \pm 0.06$ & $1.91 \pm 0.03$ & $1.75 \pm 0.08$ & 0.07 \\
\hline Diarrhea rate ${ }^{2}$ & $18.10 \pm 3.08$ & $19.94 \pm 1.79$ & $14.24 \pm 6.07$ & 0.16 \\
\hline
\end{tabular}

${ }^{1} n=6$ per treatment. All values are means \pm SEM

2 Diarrhea rate was for overall period

${ }^{a}$, b Different letters means there were statistically significant differences among three treatments when $P$-value $<0.05$ 
Table 2 Effect of combined probiotics on the immune response in serum of weaned pigs ${ }^{1}$

\begin{tabular}{lllll}
\hline Items & Control & Antibiotic & Probiotics & $P$-value \\
\hline $\operatorname{lgA}(\mathrm{g} / \mathrm{L})$ & $0.89 \pm 0.11$ & $0.81 \pm 0.07$ & $0.85 \pm 0.05$ & 0.34 \\
$\operatorname{lgG}(\mathrm{g} / \mathrm{L})$ & $7.19 \pm 1.90$ & $7.1 \pm 0.87$ & $7.3 \pm 1.34$ & 0.98 \\
$\mathrm{IL}-10(\mathrm{pg} / \mathrm{mL})$ & $60.60 \pm 6.29^{\mathrm{a}}$ & $46.91 \pm 8.42^{\mathrm{b}}$ & $62.96 \pm 1.21^{\mathrm{a}}$ & 0.02 \\
$\mathrm{IL}-1 \beta(\mathrm{pg} / \mathrm{mL})$ & $48.18 \pm 6.29^{\mathrm{a}}$ & $40.89 \pm 5.67^{\mathrm{b}}$ & $38.45 \pm 3.28^{\mathrm{b}}$ & 0.02 \\
IL-6 $(\mathrm{pg} / \mathrm{mL})$ & $92.04 \pm 8.74^{\mathrm{a}}$ & $77.89 \pm 6.61^{\mathrm{b}}$ & $72.99 \pm 9.97^{\mathrm{b}}$ & 0.02 \\
TNF-a $(\mathrm{pg} / \mathrm{mL})$ & $81.55 \pm 7.29$ & $75.80 \pm 3.89$ & $75.76 \pm 2.03$ & 0.17 \\
IFN- $(\mathrm{pg} / \mathrm{mL})$ & $64.50 \pm 8.05^{\mathrm{a}}$ & $48.45 \pm 3.83^{\mathrm{b}}$ & $32.46 \pm 8.01^{\mathrm{c}}$ & $<0.01$
\end{tabular}

${ }^{1}$ Serum samples were obtained from one pig randomly selected from each replicate, which means six pigs per group. All values are means \pm SEM $a, b, c$ Different letters means there were statistically significant differences among three treatments when $P$-value $<0.05$

reduced the serum level of interleukin-10 (IL-10) $(P<0.05)$. Probiotics and antibiotic did not affect serum concentrations of IgA, IgG, or tumor necrosis factor $\alpha$ (TNF- $\alpha) 28$ days after weaning.

\section{Sequence depth and bacterial diversity of pig intestinal microbiota}

A total of 653,050 sequences were generated from 18 digesta samples (9 caecal digesta samples and 9 colonic digesta samples) after noise sequences were discarded according to the minimum sequencing depth. At the 97\% sequence similarity, 651 operational taxonomic units (OTUs) were clustered and then allotted to 13 phyla, 21 classes, 34 orders, 57 families, 177 genera and 314 species.

The differences in intestinal bacterial diversity and richness among three groups are shown in Table 3. The probiotics group tended to decrease the caecal bacterial richness (Ace, Chao) and colonic Sobs index without

Table 3 Alpha-diversity of bacterial community in the caecal and colonic digesta of weaned pigs ${ }^{1}$

\begin{tabular}{lllll}
\hline Sample & Control & Antibiotic & Probiotics & P-value \\
\hline \multicolumn{2}{l}{ Caecal digesta } & & & \\
Sobs & $420.00 \pm 24.88$ & $364.00 \pm 39.15$ & $315.00 \pm 58.80$ & 0.08 \\
Ace & $484.93 \pm 28.01$ & $416.52 \pm 44.75$ & $366.36 \pm 58.34$ & 0.05 \\
Chao & $484.75 \pm 30.28$ & $420.03 \pm 36.42$ & $373.23 \pm 68.57$ & 0.05 \\
Shannon & $4.40 \pm 0.24$ & $3.77 \pm 0.58$ & $3.71 \pm 0.60$ & 0.18 \\
Simpson & $0.03 \pm 0.01$ & $0.07 \pm 0.06$ & $0.07 \pm 0.04$ & 0.43 \\
Colonic digesta & & & \\
Sobs & $475.33 \pm 9.07$ & $427.33 \pm 47.06$ & $381.67 \pm 34.65$ & 0.05 \\
Ace & $521.66 \pm 23.2$ & $476.79 \pm 43.89$ & $446.49 \pm 34.49$ & 0.11 \\
Chao & $529.69 \pm 30.55$ & $480.54 \pm 50.12$ & $476.30 \pm 60.38$ & 0.30 \\
Shannon & $4.62 \pm 0.07$ & $4.08 \pm 0.38$ & $3.74 \pm 0.50$ & 0.06 \\
Simpson & $0.03 \pm 0.002$ & $0.05 \pm 0.03$ & $0.09 \pm 0.06$ & 0.06 \\
\hline
\end{tabular}

${ }^{1}$ Caecal and colonic digesta samples were obtained from three pigs per group and their microbiota composition were analyzed using 16S rRNA sequencing. All values are means \pm SEM affecting bacterial diversity compared to control group and antibiotic group. In addition, probiotics group had a decreased trend in bacterial community richness (Sobs) and the bacterial diversity (Shannon, Simpson) of the colonic digesta. The bacterial diversity and richness of antibiotic group fell in between the control group and probiotics group.

For beta-diversity analysis, PCoA based on Bray-Curtis distances was performed in caecal and colonic microbiota collected from pigs of the three groups. The result (Fig. 1) showed that the microbiota from pigs of probiotics group was separated from those in the control and antibiotic groups.

\section{Core bacteria of weaned pigs}

At the phylum level, Firmicutes, Bacteroidetes, and Proteobacteria were dominating in caecal and colonic digesta in all three groups, which included $99 \%$ of taxa. Spirochaetae, rich in the control group, was nearly undetectable in the probiotics and antibiotic groups (Fig. 2). At genus level, Lactobacillus (27.30\%) was more abundant in the caecal digesta of piglets fed combined L. fermentum and P. acidilactici than that in control (10.48\%) and antibiotic groups (4.07\%). Similarly, in the colonic digesta, Lactobacillus was more abundant in probiotics group (39.90\%) than in the control $(8.36 \%)$ and antibiotic groups (10.51\%) (Fig. 3). Prevotellaceae_NK3B31_group, Lactobacillus and Streptococcus were the most abundant three genera in both caecal and colonic digesta in the control group, while Clostridium sensu_stricto_1 was common dominant of the antibiotic group in both caecal and colonic digesta.

\section{Changes in microbial composition after probiotics administration}

To clearly identify how the bacteria changed among the three treatments, the linear discriminant analysis (LDA) effect size (LEfSe) were used to evaluate the differences in the relative abundance from phylum to genus (Fig. 4).

In the caecal digesta, proportion from the Lactobacillaceae family to Lactobacillus genus was significantly increased by combined L. fermentum and P. acidilactici supplementation, while greater relative abundance of Treponema_2 from Spirochaetae phylum, Anaerovibrio genus and two strains from Lachnospiraceae were observed in the control group. Besides, the abundance of Anaerofilum, Turicibacter, Rothia and Intestinibacter genus were increased significantly in the antibiotic group.

In the colon, the abundance of Firmicutes phylum was higher in probiotics group compared to the control group, while the relative abundance of Anaerofilum, Prevotella_1 from Bacteroidetes phylum and Succinivibrio from Aeromonadales order were lower. 


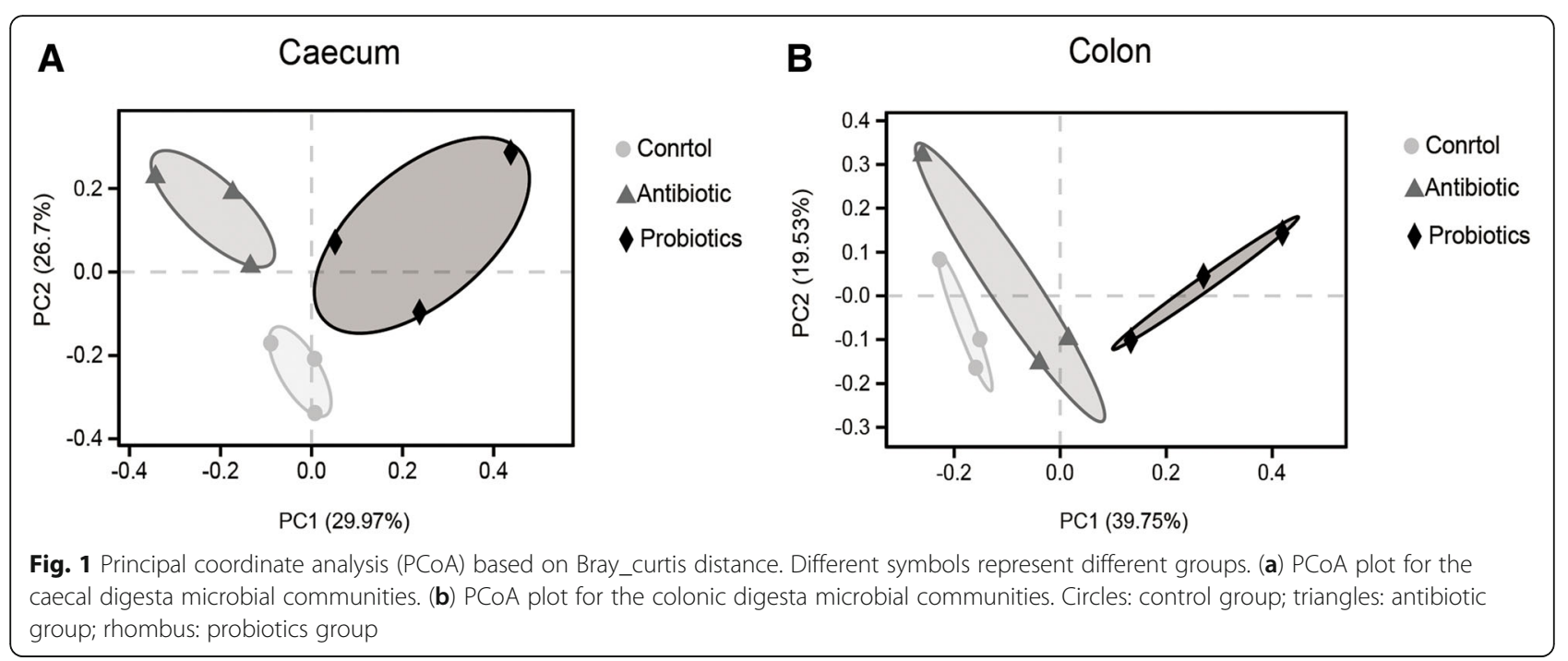

\section{SCFAs concentrations}

The concentrations of 4 short chain fatty acids in the caecal and colonic digesta of pigs were measured (Table 4). Compared with the other two groups, acetic acid and propionic acid in caecal digesta of probiotics fed pig were higher $(P<0.05)$. No significant differences in caecal lactic acid and butyric acid were detected among the three groups. As for the concentrations of SCFAs in colonic contents, contents of lactic acid and propionic acid of the antibiotic group were significantly lower than the other two groups $(P<0.05)$. There was no statistical difference in the colonic acetic acid and butyric acid among the three groups.

\section{Discussion}

Probiotics, when administered in proper proportion, can modulate the intestinal microbial population, reduce diarrhea and boost the immune system of the host, which can then enhance gut health and growth performance of pigs
$[13,14]$. There have been some previous studies focusing on identifying the beneficial effect of individual probiotic specie $L$. fermentum and $P$. acidilactici fed individually as probiotics for animals $[8,9,11,12]$. However, whether the combined $L$. fermentum and P. acidilactici could complement individual effects to each other and modulate gut bacterial community better have not been studied. Therefore, we tried to understand the effects of dietary supplementation of combined $L$. fermentum and P. acidilactici preparation on weaned pigs in the present study. Weaned piglets were fed a basal diet, or supplemented with chlortetracycline or combined $L$. fermentum and $P$. acidilactici in the current study. Our results showed that supplementing diets with probiotics could improve growth performance, regulate inflammation through lowering proinflammatory factors, and change the composition of intestinal microbiota.

Weaning may be accompanied with diarrhea and growth inhibition in piglets [2], growth performance is

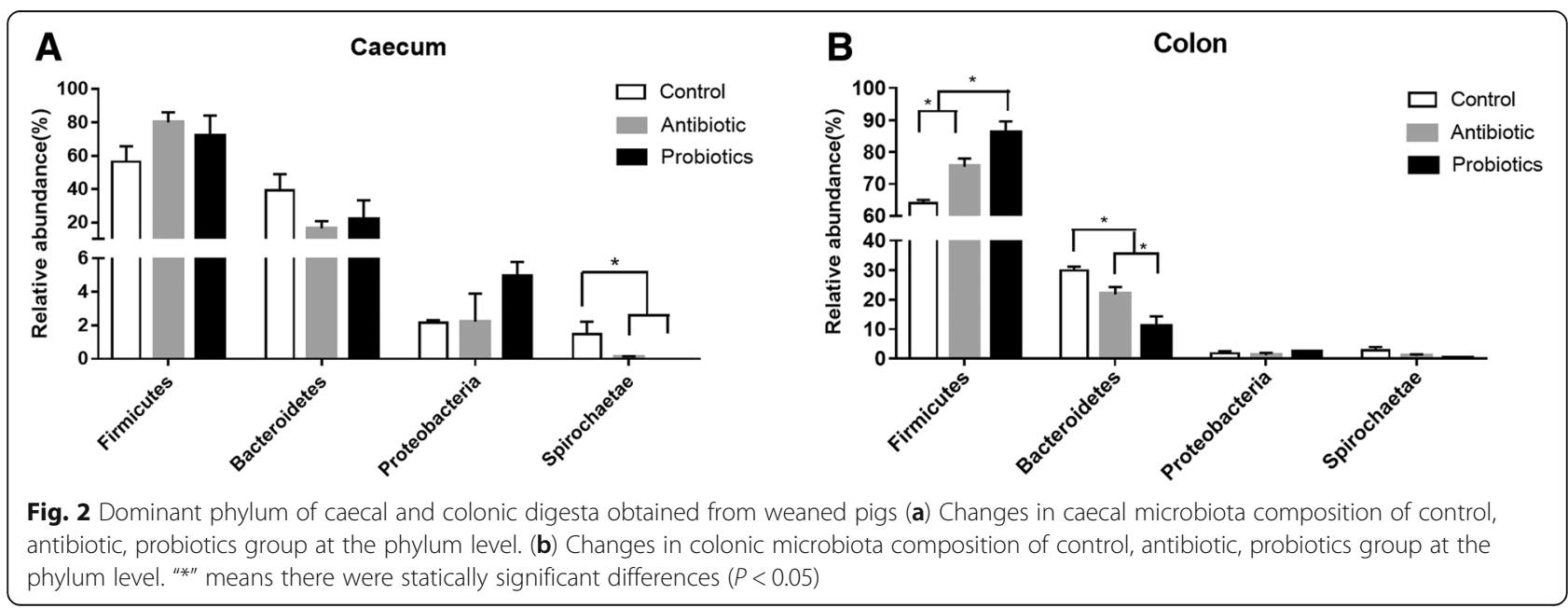




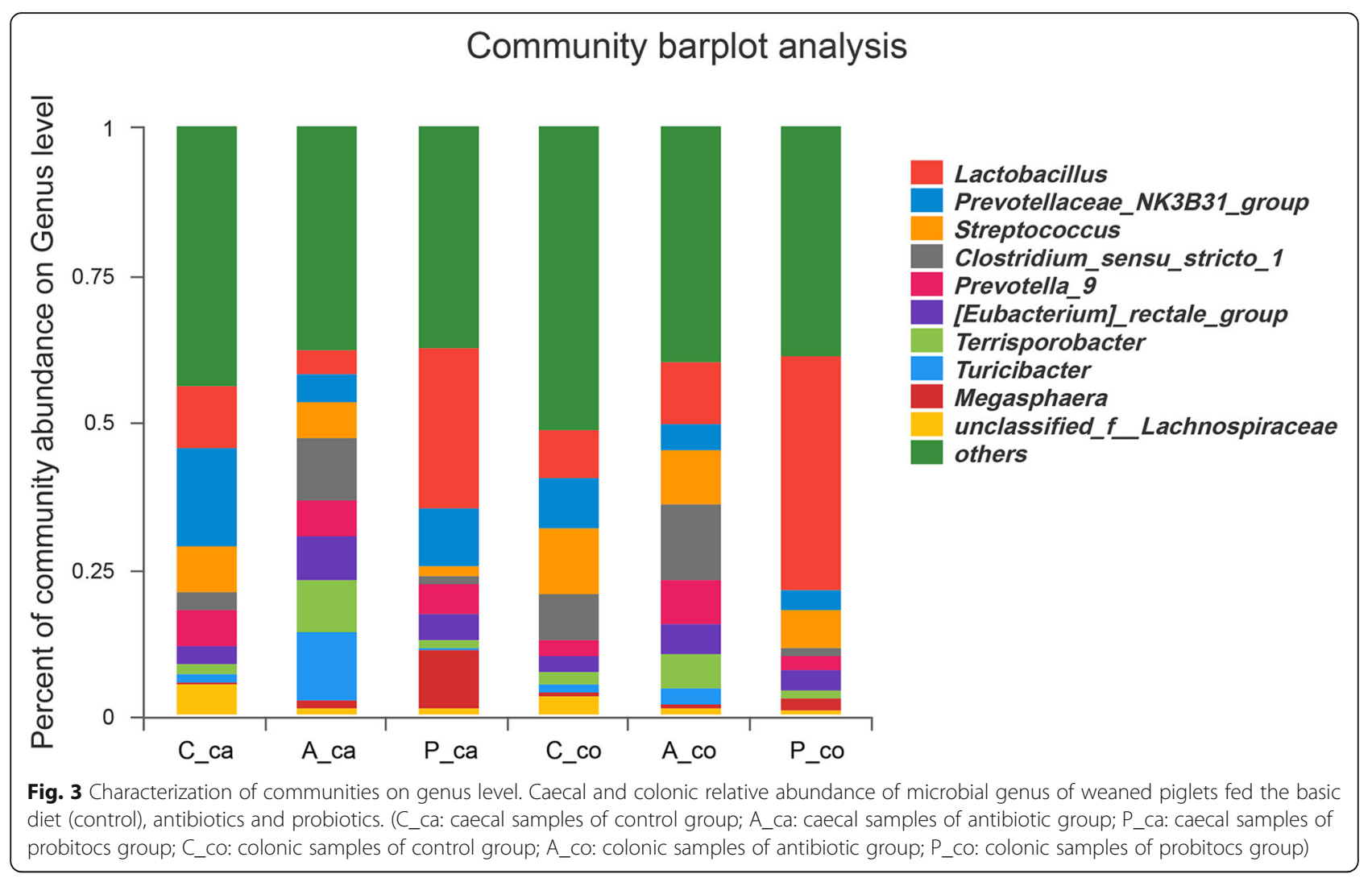

one of the most important aspects of animal performance in the pig industry. In this study, we observed that weaned pigs supplemented with combined $L$. fermentum and $P$. acidilactici preparation demonstrated greater ADG for the whole 28 days and improved feed efficiency in the latter half of the trial compared with weaned pigs in the other two groups. A previous study identified that administration of Pediococcus acidilactici FT28 enhanced feed conversion rate of weaned piglets compared with the control group [15], which corresponds with our result but it had no significant effect on the ADG. Our finding is also supported by earlier research that weaned pigs supplemented with probiotic complexes containing Enterococcus faecium, Bacillus subtilis and Lactobacillus paracasei had greater ADG and improved feed efficiency [16]. Therefore, it can be speculated that combined probiotics exhibited additional benefits on growth performance compared with a single probiotic specie. Besides, $L$. fermentum 15007 was identified in enhancing the levels of proteins in relation to energy metabolism and protein synthesis of weaned piglets, whereas chlortetracycline reduced them [10], which may account for the different growth performance between the probiotics group and the antibiotic group.

Growth performance of animals is also relevant to their health status and immunity. Both probiotics group and antibiotic group were followed by the reduction in the serum concentrations of IL- 6 and IFN- $\gamma$ compared with control group. Probiotics group uniquely decreased IL-1 $\beta$ compared with the other two groups. The proinflammatory cytokines IL- $1 \beta$ and IL- 6 are significant in the immune response and the tissue maintenance [1719]. In agreement with this study, it was reported that Lactobacillus reuteri and L. fermentum could depress the expression of pro-inflammatory factor TNF- $\alpha$ in a rat colitis model [20]. In another study, the control group not receiving probiotics had an increased expression of pro-inflammatory cytokines [21]. Interestingly, the anti-inflammatory cytokine, IL-10 in serum, was reduced in the antibiotic group. The IL-10 is an important immune regulator in the gut tract, which is mainly released by B lymphocytes [22]. All in all, probiotics helped to regulate the inflammatory response, reduce inflammatory damage and then improve health of weaned pigs, which performed better than antibiotic fed pigs.

When it comes to the intestinal microbiota, we mainly focused on the microbiota from caecal and colonic digesta of the weaned pigs where microbial population is abundant and diverse [13]. Our results showed that combined $L$. fermentum and $P$. acidilactici tended to decrease the alpha diversity of intestinal microbiota in a similar trend with antibiotic, which may be due to the fact that probiotics can inhibit pathogens and modulate intestinal microbiota. 


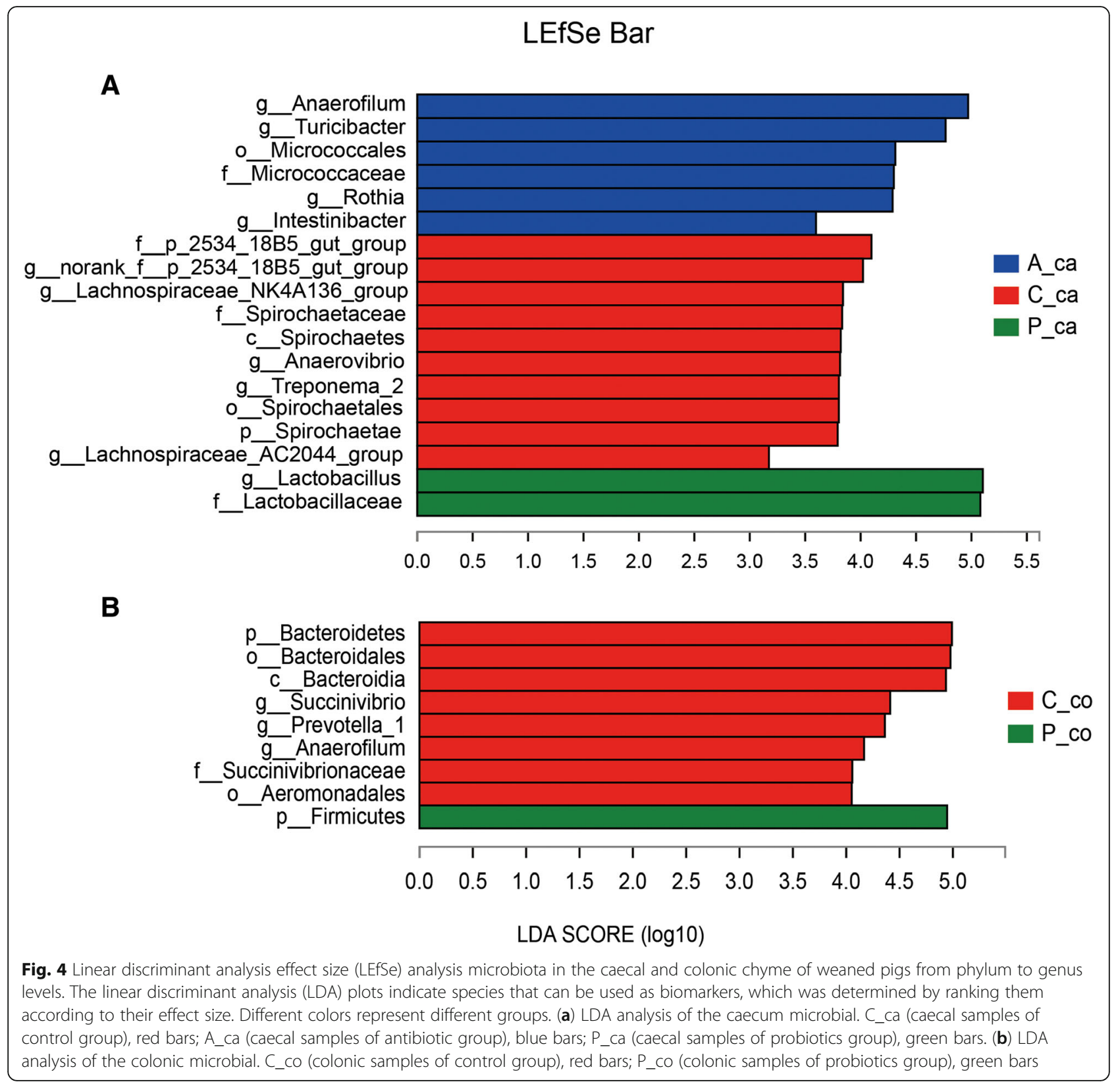

Firmicutes, Bacteroidetes, Proteobacteria and Spirochaetae were the top four phyla in both caecal and colonic digesta. These results were consistent with a previous study [23], where Firmicutes and Bacteroidetes were most dominant. In the caecal digesta, the relative abundance of Treponema_2 genus and its phylum Spirochaetae were significantly abundant in the control group than the probiotics and antibiotic groups. Spirochaetae was nearly undetectable in the other two groups, similar to a previous study in which pigs were fed Lactobacillus salivarius UCC118 WT [24]. One genus of the spirochetes was identified to be related to swine diarrhea [25]. Thus the ability of the combined $L$. fermentum and
P. acidilactici to control destructive bacteria could be significant, which may benefit growth. In colonic digesta, we found that both the probiotics group and antibiotic group significantly increased the abundance of Firmicutes and decreased the abundance of Bacteroidetes. These results were consistent with a previous study which showed that establishment of Firmicutes in ileal microbiota of weaned pigs was promoted by $P$. acidilactici and antibiotic treatment [11]. The phyla Firmicutes and Bacteroidetes are known for polysaccharide fermentation. When intestinal Firmicutes / Bacteroidetes ratio was improved, the host are able to absorb more energy from the diet and the ability to store energy are strengthened [26, 
Table 4 Concentrations of short chain fatty acids in the caecal and colonic digesta of weaned pigs ${ }^{1}$

\begin{tabular}{|c|c|c|c|c|}
\hline Item & Control & Antibiotic & Probiotics & $P$-value \\
\hline \multicolumn{5}{|l|}{ Caecal digesta } \\
\hline lactic acid (mg/g) & $2.66 \pm 0.52$ & $2.57 \pm 0.63$ & $2.91 \pm 0.03$ & 0.67 \\
\hline Acetic acid (mg/g) & $5.29 \pm 0.58^{b}$ & $5.32 \pm 0.28^{b}$ & $6.23 \pm 0.49^{a}$ & 0.03 \\
\hline Propionic acid (mg/g) & $3.02 \pm 0.70^{b}$ & $2.92 \pm 0.24^{b}$ & $3.73 \pm 0.45^{\mathrm{a}}$ & 0.02 \\
\hline Butyric acid (mg/g) & $1.32 \pm 0.39$ & $1.75 \pm 0.48$ & $1.54 \pm 0.41$ & 0.34 \\
\hline \multicolumn{5}{|l|}{ Colonic digesta } \\
\hline lactic acid (mg/g) & $5.30 \pm 0.54^{\mathrm{a}}$ & $3.87 \pm 0.66^{b}$ & $5.60 \pm 0.53^{a}$ & 0.01 \\
\hline Acetic acid (mg/g) & $5.93 \pm 0.33$ & $5.92 \pm 0.48$ & $6.49 \pm 0.59$ & 0.21 \\
\hline Propionic acid (mg/g) & $3.02 \pm 0.41^{\mathrm{a}}$ & $2.53 \pm 0.30^{b}$ & $3.07 \pm 0.30^{\mathrm{a}}$ & 0.04 \\
\hline Butyric acid (mg/g) & $1.54 \pm 0.22$ & $1.76 \pm 0.28$ & $1.60 \pm 0.25$ & 0.47 \\
\hline
\end{tabular}

27]. The existence of the common greater beneficial phyla and the less potential pathogen in the probiotics group may be potential factors involved in the increased body weight gain in the probiotics group.

Lactobacillus, Prevotellaceae_NK3B31_group, Streptococcus and Clostridium_sensu_stricto_1 were the dominant genera in three groups, but the most dominant genera were different in each group. Lactobacillaceae family and Lactobacillus genus did affect the caecal microbiota of the probiotics group. Lactobacillus can produce energy through glycolysis and ferment carbohydrates into lactic acid without oxygen [28]. Although there was no statistical difference in lactic acid concentration of caecal digesta, it may contribute to the higher SCFAs (acetic acid and propionic acid) levels in the caecal digesta. Similar results were showed in a previous study about probiotic complexes containing Enterococcus faecium, Bacillus subtilis and Lactobacillus paracasei [16]. Meanwhile, the Lactobacillus level tended to improve in the colonic digesta, which partly explained higher lactic acid and propionic acid than the antibiotic group. Some strains of Lactobacillus have been found possess anti-bacterial activities, antiinflammation [29], and increased Lactobacillus in the intestinal digesta [30]. The Lactobacillus promoted the production of SCFAs, suppressed pathogens with an acidic environment and digested nutrients that the host cannot metabolize, which ultimately improves intestinal health status and growth.

In addition, 5 genera in the caecal digesta and 3 genera in the colonic digesta were more abundant in the control group compared to the other two groups. Of these unique genera, Anaerovibrio, an anaerobic bacteria belonging to the Negativicutes class, was higher in the control group. The members of Negativicutes have a peculiar cell wall composition which is Gram negative [31]. Interestingly, lactic acid produced by Lactobacillus is vital to antimicrobial activities for suppressing the generation and growth of Gram-negative bacteria virulence factors [32, 33]. The combined L. fermentum and $P$. acidilactici may decrease the Anaerovibrio by promoting lactic acid production, which permeated the gramnegative bacterial outer membrane [34]. The reduction of pathogenic microbial load in intestines could limit the negative influence of microbiota towards its host, which may improve growth performance of pigs [35]. In the colonic digesta, the high abundance Prevotella_1 and Succinivibrio genus in control group are the producer of acetic acids and succinic acids, which were end-products of fermentation [36]. Succinivibrio was sensitive to antibiotics [37], which partly explains the lower colonic lactic acid and propionic acid in the antibiotic group.

In the antibiotic group, caecal bacterial abundance of 4 genera increased compared to the other two groups. For example, the abundance of Rothia genus and its order Micrococcales were higher. Rothia spp. cause extensive severe infections, particular in immunocompromised hosts [38], which may be related to lower serum IL-10 level. All in all, the antibiotic modulated the intestinal microbiota by inhibiting specific bacteria, but there were still potentially harmful bacteria, owning to its disturbance of the normal balance of gut microflora. Therefore, the growth performance of antibiotic group was not as good as that of the probiotics group, which also provided information that the combined $L$. fermentum and $P$. acidilactici has potential preventive effect like antibiotic and could improve pig performance.

A potential limitation of this study is that the sample size of sacrificed pigs for the SCFAs concentrations and gut microbiome analysis is a little small, but some tests indicated a difference among three groups. We will carry out a larger sample study in the future and focus on the SCFAs concentrations and gut microbiome analysis test. 


\section{Conclusions}

In conclusion, dietary supplementation with combined $L$. fermentum and P. acidilactici improved ADG and F/G of weaned pigs. Concentrations of the serum proinflammatory factors IL-6, IFN- $\gamma$ were significantly decreased in the probiotics group and antibiotic group compared with the control group. In addition, the probiotics group enriched the abundance of Lactobacillus in the caecal digesta and Firmicutes in the colonic digesta, which may be related to greater concentrations of caecal SCFAs in the probiotics group. In addition, the growth of Treponema_2, Anaerovibrio in the caecal digesta was inhibited in the probiotics and antibiotic groups. These findings suggest that combined Lactobacillus fermentum and Pediococcus acidilactici supplementation of diets improved growth performance, alleviated inflammation and regulated gut health by promoting beneficial bacteria, inhibiting pathogens, and promoting the production of SCFAs in weaned pigs.

\section{Methods}

\section{Animals, diets and experimental design}

Animal protocols were conducted on the basis of the regulations of China Agricultural University Animal Care and Use Committee (Beijing, China). The weaned pigs were owned by FengNing Swine Research Unit of China Agricultural University (Academician Workstation in Chengdejiuyun Agricultural and Livestock Co., Ltd).

One hundred and eight pigs (Duroc $\times$ Landrace $\times$ Large White) were weaned at $28 \mathrm{~d}$ of age $(7.12 \pm 0.08 \mathrm{~kg})$, were divided into three groups with randomized design considering the litter of origin and gender. Piglets from the same litter were assigned to different treatment group. There were 6 replicate pens per treatment group and 6 pigs per pen (half male and half female). There was no statistical difference among the average initial weight of weaned pigs in each group $(P=0.99)$. The animal size was based on a published study [39]. The experimental treatments were as follows (Additional file 1: Table S1): i. Control: basal diet; ii. Antibiotic: the basal diet plus $75 \mathrm{mg} \cdot \mathrm{kg}^{-1}$ chlortetracycline (commercially available chlortetracycline with a purity of 15\%); and iii. Probiotics: basal diet plus $4 \%$ compound probiotics. The different diets were started to be supplied to the pigs weaned at $28 \mathrm{~d}$ of age and the experimental period lasting for 28 days. Pigs were fed in a nursery house with plastic leakage dung floors. Water and feed were supplied ad libitum during the experimental period.

\section{Probiotics compound preparation}

The L. fermentum and P. acidilactici-containing probiotics compound preparation was provided by Hebei Daguang Biotechnology Co. LTD, which is a fermented feed additive. The combined probiotics preparation were cultured and fermented into liquid, then the fermentation substrates including $60 \%$ wheat bran, $10 \%$ corn meal, and $30 \%$ soybean meal were added and the solidliquid combinations were fermented. The combined probiotics preparation was stored in the feed fermentation bag with single exhaust valve for maintaining its natural high activity. The final counted viable bacteria was $1.6 \times$ $10^{9} \mathrm{CFU} / \mathrm{g}$, mainly including $9.1 \times 10^{8} \mathrm{CFU} / \mathrm{g}$ Lactobacillus fermentum and $5.25 \times 10^{8} \mathrm{CFU} / \mathrm{g}$ Pediococcus acidilactici. The pigs were fed four times per day at 8:30, 11: 30, 14:30 and 17:30. The compound probiotics preparation was mixed with feed in a proportion of $4 \%$ just before feeding the pigs.

\section{Sample collection}

All pigs were weaned at the $28 \mathrm{~d}$ of age and the diet treatment started from weaning day and lasted for 28 days. During the experiment period, pig were weighed individually at d 1, d 14 and d 28 after weaning. Feed consumption of each pen was monitored. Average daily gain (ADG), average daily feed intake (ADFI) and feed consumed/weight gain (F/G) during $\mathrm{d} 1$ to $\mathrm{d} 14$ and $\mathrm{d}$ 14 to $\mathrm{d} 28$, were calculated to observe the growth performance of piglets. And the diarrhea rate was calculated based on the fecal score system as follows: 0, normal consistency; 1, pasty; 2, semiliquid; and 3 liquid. Pigs with a score of 2 or greater were considered to have diarrhea [40]. Diarrhea rate $(\%)=100 *$ [total number of diarrhea cases in each pen during the trial period / (total number of pigs in each pen * total number of days in the trial)].

On the $\mathrm{d} 28$, one pig was selected randomly from each replicate pen to collect blood samples from the jugular vein. After blood samples were naturally coagulated, they were immediately centrifuged at $3000 \mathrm{~g}$ for $10 \mathrm{~min}$, and then removed and placed in $-20{ }^{\circ} \mathrm{C}$ freezer to be analyzed for immunoglobulins and inflammatory cytokines.

Three pigs per treatment were randomly selected. The selected pigs were from the different pens and their body weights were representative of the average body weights. The sample size for intestinal microbiota analysis was based on a related study [3]. The pigs were sacrificed after exsanguination under anesthesia by intravenous injection with sodium pentobarbital $(40 \mathrm{mg} / \mathrm{kg} \mathrm{BW})$ in a slaughter house. Approximately $10 \mathrm{~g}$ digesta samples were collected into sterile tubes from the mid colon and caecum of each pig. Digesta samples were stored in liquid nitrogen immediately before being removed to the laboratory and stored at $-80^{\circ} \mathrm{C}$ freezer until the analysis of short chain fatty acids (SCFAs) concentrations and microbiota composition. The investigators were blinded to group allocation during the experiments analysis. 
Analysis of immunoglobulins and inflammatory cytokines of serum samples

Serum concentrations of IgA (144118001; Mindray Co., Ltd., Shenzhen; China) and IgG (14438002; Mindray Co., Ltd., Shenzhen; China) on day 28 were analyzed by a Hitachi 7600 automatic biochemical analyzer. The levels of interleukins interleukin-1 $\beta$ (IL-1 $\beta$ ), interleukin-10 (IL10) and interleukin-6 (IL-6), interferon- $\gamma$ (IFN- $\gamma$ ), and tumor necrosis factor (TNF- $\alpha)$ were measured using commercial ELISA kits for pigs following the manufacturer's instructions (Konka Hongyuan Biotechnology Co., Ltd., Beijing; China).

\section{Microbial analysis}

\section{DNA extraction and PCR amplification and sequencing}

Microbial DNA was extracted from digesta using the QIAamp R Fast DNA Stool Mini Kit (Qiagen Ltd., Germany) according to manufacturer's instructions. The final DNA quality were examined by $1 \%$ agarose gel electrophoresis and NanoDrop 2000 UV-vis spectrophotometer (Thermo Scientific, Wilmington, USA). The V3-V4 regions of the bacteria $16 \mathrm{~S}$ rRNA gene were generated with universal primers 338F (5'-ACTCCTACGGGAGG CAGCAG-3') and 806R (5'-GGACTACHVGGGTWTC TAAT-3') by PCR amplification [41]. The PCR reactions were conducted as follows: denaturation at $95^{\circ} \mathrm{C}$ for 3 min, 27 cycles at $95^{\circ} \mathrm{C}$ for $30 \mathrm{~s}, 30 \mathrm{~s}$ for annealing at $55^{\circ} \mathrm{C}$, and elongation at $72{ }^{\circ} \mathrm{C}$ for $45 \mathrm{~s}$, and a final extension at $72{ }^{\circ} \mathrm{C}$ for $10 \mathrm{~min}$. Purified amplicons were pooled in equimolar and paired-end sequenced on the Illumina MiSeq platform (Illumina, San Diego, USA) [42]. All the raw data were uploaded into NCBI Sequence Read Archive database with accession number PRJNA495019. The raw sequencing data were processing in accordance with a previous study [41].

OTUs were clustered with a 97\% similarity cutoff using UPARSE(version 7.1 http://drive5.com/uparse/) and chimeric sequences were identified and removed using UCHIME. The taxonomy of each $16 \mathrm{~S}$ rRNA gene sequence was analyzed by RDP Classifier algorithm (http://rdp.cme.msu.edu/) against the Silva 128/16s_bacterial database (http://www.arb-silva.de) using confidence threshold of $70 \%$. The alpha-diversity including richness, diversity and coverage based on the Sobs, Chao, Ace, Shannon, Simpson and Coverage index within each sample were generated by mothur (version v.1.30.1). Principal coordinate analysis (PCoA) were calculated based on the Bray-Curtis distances.

\section{Digesta SCFAs analysis}

The concentrations of SCFAs in the digesta sample of each pig were analyzed in the high-performance ion chromatography system (DIONEX ICS-3000; Thermo Fisher, Waltham, MA; USA) [43]. Approximately $0.5 \mathrm{~g}$ of digesta content was weighed into a $10 \mathrm{ml}$ centrifuge tube. Each sample was weighed for two tubes. Ultrapure water $(8 \mathrm{ml})$ was added and then vortexed to mix the contents. After centrifugation at $4000 \mathrm{r} / \mathrm{min}$ for $15 \mathrm{~min}$, $0.16 \mathrm{ml}$ supernatant was removed into a $10 \mathrm{ml}$ centrifuge tube, and $7.84 \mathrm{ml}$ of ultrapure water solution (equivalent to a 50-fold dilution) was added. Then filtered the dilution with a $0.22 \mu \mathrm{m}$ membrane and analyzed (Dionex IonPac AS11-HC; Thermo Fisher, Waltham, MA; USA). The concentrations of short chain fatty acids were calculated.

\section{Statistical analysis}

The data of growth performance, inflammatory cytokines, and SCFAs were subjected to an analysis of variance method using SAS software (Windows V8) with general linear model procedure, comparing the differences among three groups. Six pigs in one pen were considered as the experimental unit of analyses for the difference in growth performance. As for inflammatory cytokines, SCFAs and microbial analysis, individual pigs were considered as the experimental unit. Outliers were eliminated based on $3 \delta$ criterion. Significant differences among means of groups were determined by Duncan's multiple range tests. $P$-values $<0.05$ were considered as statistically significant. All values are presented as means \pm SEM. The difference in the alpha diversity among three groups was tested using Kruskal-Wallis (KW) test (SAS Windows V8) and P-values were adjusted with FDR when they were $<5 \%$. LEfse was used to conduct linear discriminant analysis (LDA) to estimate the effect of abundance of each component (species) on the differences. And the multi-group comparison strategy was allagainst-all.

\section{Additional file}

Additional file 1: Table S1. Diet composition and nutrient levels ${ }^{1}$ (DOCX $16 \mathrm{~kb})$

\section{Abbreviations \\ ADFI: Average daily feed intake; ADG: Average daily gain; F/G: Feed consumed/weight gain; IFN- $\gamma$ : Interferon- $\gamma$; IL-10: Interleukin-10; IL- \\ 1 $\beta$ : Interleukin-1 $\beta$; IL-6: Interleukin-6; LDA: Linear discriminant analysis; LEfSe: Linear discriminant analysis effect size; OTUs: Operational taxonomic units; PCoA: Principal coordinate analysis; SCFAs: Short chain fatty acids; TNF- a: Tumor necrosis factor-a}

\footnotetext{
Acknowledgements

The authors thank FengNing Swine Research Unit of China Agricultural University (Academician Workstation in Chengdejiuyun Agricultural and Livestock Co., Ltd) and Mr. Feng Zhang for the supports in the pig experiment. We thank Lee Johnston for the English edition of the manuscript.
}

Consent to publication

Not applicable. 


\section{Authors' contributions}

SW carried out the experiment, analyzed samples and statistical data and wrote the manuscript. BY and HG participated in the animal experiment. ST and $\mathrm{SZ}$ contributed to revise the manuscript. SH and BH contributed to the analysis of some samples and interpreted some results. JZ and JW were corresponding authors, who mainly designed the study and supervised the whole program. All authors have read and approved the final manuscript.

\section{Funding}

This work was supported by National Key Research and Development Program of China (2016YFD0500506), the National Undergraduate Innovation Project (201710019079), the Beijing Municipal Natural Science Foundation (No. S170001), the National Natural Science Foundation of China (31630074), the 111 Project (B16044), Jinxinnong Animal Science Developmental Foundation and the Hunan Co-Innovation Centre of Animal Production Safety, CICAPS. They financially supported analysis fees.

\section{Availability of data and materials}

The 16S rRNA gene sequences raw datasets generated during the current study are available in the NCBI Sequence Read Archive (SRA) database with accession number PRJNA495019. The diet composition and nutrient levels are included within its supplementary information files. The other datasets used during the current study are available from the corresponding author on reasonable request.

\section{Ethics approval and consent to participate}

Animal protocols were conducted on the basis of the regulations of China Agricultural University Animal Care and Use Committee (Beijing, China). The experiment was carried out in FengNing Swine Research Unit of China Agricultural University (Academician Workstation in Chengdejiuyun Agricultural and Livestock Co., Ltd)

\section{Competing interests}

The authors declare that they have no competing interests.

Received: 29 November 2018 Accepted: 2 July 2019

Published online: 10 July 2019

\section{References}

1. Castillo M, Martin-Orue SM, Nofrarias M, Manzanilla EG, Gasa J. Changes in caecal microbiota and mucosal morphology of weaned pigs. Vet Microbiol. 2007;124:239-47.

2. Li PH, Niu Q, Wei QT, Zhang YQ, Ma X, Kim SW, Lin MX, Huang RH. Microbial shifts in the porcine distal gut in response to diets supplemented with Enterococcus Faecalis as alternatives to antibiotics. Sci Rep. 2017;7: 41395.

3. Li K, Xiao Y, Chen J, Chen J, He X, Yang H. Microbial composition in different gut locations of weaning piglets receiving antibiotics. AsianAustralas J Anim Sci. 2017:30:78-84.

4. Gresse R, Chaucheyras-Durand F, Fleury MA, Van de Wiele T, Forano E, Blanquet-Diot S. Gut microbiota Dysbiosis in Postweaning piglets: understanding the keys to health. Trends Microbiol. 2017;25:851-73.

5. Liu H, Ji HF, Zhang DY, Wang SX, Wang J, Shan DC, Wang YM. Effects of Lactobacillus brevis preparation on growth performance, fecal microflora and serum profile in weaned pigs. Livest Sci. 2015;178:251-4.

6. Jiao LF, Song ZH, Ke YL, Xiao K, Hu CH, Shi B. Cello-oligosaccharide influences intestinal microflora, mucosal architecture and nutrient transport in weaned pigs. Anim Feed Sci Technol. 2014;195:85-91.

7. Schwarzer M, Makki K, Storelli G, Machuca-Gayet I, Srutkova D, Hermanova P, Martino ME, Balmand S, Hudcovic T, Heddi A, Rieusset J, Kozakova H, Vidal $\mathrm{H}$, Leulier F. Lactobacillus plantarum strain maintains growth of infant mice during chronic undernutrition. Science. 2016;351:854-7.

8. Wang AN, Cai CJ, Zeng XF, Zhang FR, Zhang GL, Thacker PA, Wang JJ, Qiao SY. Dietary supplementation with Lactobacillus fermentum 15007 improves the anti-oxidative activity of weanling piglets challenged with diquat. J Appl Microbiol. 2013;114:1582-91.

9. Davoodabadi A, Dallal MMS, Lashani E, Ebrahimi MT. Antimicrobial activity of Lactobacillus spp. isolated from fecal Flora of healthy breast-fed infants against Diarrheagenic Escherichia coli. Jundishapur Journal of Microbiology. 2015;8:e27852
10. Wang X, Yang F, Liu C, Zhou H, Wu G, Qiao S, Li D, Wang J. Dietary supplementation with the probiotic Lactobacillus fermentum 15007 and the antibiotic aureomycin differentially affects the small intestinal proteomes of weanling piglets. J Nutr. 2012;142:7-13.

11. Brousseau JP, Talbot G, Beaudoin F, Lauzon K, Roy D, Lessard M. Effects of probiotics Pediococcus acidilactici strain MA18/5M and Saccharomyces cerevisiae subsp. boulardii strain SB-CNCM I-1079 on fecal and intestinal microbiota of nursing and weanling piglets. J Anim Sci. 2015;93:5313-26.

12. Wang JQ, Yin FG, Zhu C, Yu H, Niven SJ, de Lange CFM, Gong J. Evaluation of probiotic bacteria for their effects on the growth performance and intestinal microbiota of newly-weaned pigs fed fermented high-moisture maize. Livest Sci. 2012;145:79-86.

13. Chaucheyras-Durand F, Durand $\mathrm{H}$. Probiotics in animal nutrition and health. Benef Microbes. 2010;1:3-9.

14. Liao SF, Nyachoti M. Using probiotics to improve swine gut health and nutrient utilization. Anim Nutr. 2017;3:331-43.

15. Dowarah R, Verma AK, Agarwal N, Singh P, Singh BR. Selection and characterization of probiotic lactic acid bacteria and its impact on growth, nutrient digestibility, health and antioxidant status in weaned piglets. PLoS One. 2018;13:e0192978.

16. Lu X, Zhang M, Zhao L, Ge K, Wang Z, Jun L, Ren F. Growth performance and post-weaning diarrhoea in piglets fed a diet supplemented with probiotic complexes. Journal. 2018. https://doi.org/10.4014/jmb.1807.07026.

17. Ligumsky M, Simon PL, Karmeli F, Rachmilewitz D. Role of interleukin 1 in inflammatory bowel disease--enhanced production during active disease. Gut. 1990;31:686-9.

18. Ishihara K, Hirano T. IL-6 in autoimmune disease and chronic inflammatory proliferative disease. Cytokine Growth Factor Rev. 2002;13:357-68.

19. Splichal I, Fagerhol MK, Trebichavsky I, Splichalova A, Schulze J. The effect of intestinal colonization of germ-free pigs with Escherichia coli on calprotectin levels in plasma, intestinal and bronchoalveolar lavages. Immunobiology. 2005:209:681-7.

20. Peran L, Sierra S, Comalada M, Lara-Villoslada F, Bailon E, Nieto A, Concha A, Olivares M, Zarzuelo A, Xaus J, Galvez J. A comparative study of the preventative effects exerted by two probiotics, Lactobacillus reuteri and Lactobacillus fermentum, in the trinitrobenzenesulfonic acid model of rat colitis. Br J Nutr. 2007;97:96-103.

21. Ermolenko E, Rybalchenko O, Borshev Y, Tarasova E, Kramskaya T, Leontieva G, Kotyleva M, Orlova O, Abdurasulova I, Suvorov A. Influence of monostrain and multistrain probiotics on immunity, intestinal ultrastructure and microbiota in experimental dysbiosis. Benef Microbes. 2018. https://doi. org/10.3920/bm2017.0117:1-14

22. Kuhn R, Lohler J, Rennick D, Rajewsky K, Muller W. Interleukin-10-deficient mice develop chronic enterocolitis. Cell. 1993;75:263-74.

23. Zhang D, Ji H, Liu H, Wang S, Wang J, Wang Y. Changes in the diversity and composition of gut microbiota of weaned piglets after oral administration of Lactobacillus or an antibiotic. Appl Microbiol Biotechnol. 2016;100:10081-93.

24. Riboulet-Bisson E, Sturme MH, Jeffery IB, O'Donnell MM, Neville BA, Forde BM, Claesson MJ, Harris H, Gardiner GE, Casey PG, Lawlor PG, O'Toole PW, Ross RP. Effect of Lactobacillus salivarius bacteriocin Abp118 on the mouse and pig intestinal microbiota. PLoS One. 2012;7:e31113.

25. Burrough ER. Swine dysentery: Etiopathogenesis and diagnosis of a reemerging disease. Vet Pathol. 2017;54:22-31.

26. Backhed F, Ding H, Wang T, Hooper LV, Koh GY, Nagy A, Semenkovich CF, Gordon JI. The gut microbiota as an environmental factor that regulates fat storage. Proc Natl Acad Sci U S A. 2004;101:15718-23.

27. Ley RE, Backhed F, Turnbaugh P, Lozupone CA, Knight RD, Gordon Jl. Obesity alters gut microbial ecology. Proc Natl Acad Sci U S A. 2005;102: 11070-5.

28. Mikkelsen LL, Naughton PJ, Hedemann MS, Jensen BB. Effects of physical properties of feed on microbial ecology and survival of Salmonella enterica serovar Typhimurium in the pig gastrointestinal tract. Appl Environ Microbiol. 2004:70:3485-92.

29. Chiang ML, Chen HC, Chen KN, Lin YC, Lin YT, Chen MJ. Optimizing production of two potential probiotic lactobacilli strains isolated from piglet feces as feed additives for weaned piglets. Asian-Australas J Anim Sci. 2015; 28:1163-70.

30. Huang CH, Qiao SY, Li DF, Piao XS, Ren JP. Effects of Lactobacilli on the performance, diarrhea incidence, VFA concentration and gastrointestinal microbial flora of weaning pigs. Asian Australas J Anim Sci. 2004;17:401-9. 
31. Sutcliffe IC. A phylum level perspective on bacterial cell envelope architecture. Trends Microbiol. 2010;18:464-70.

32. Pace RM, Prince AL, Ma J, Belfort BDW, Harvey AS, Hu M, Baquero K, Blundell P, Takahashi D, Dean T, Kievit P, Sullivan EL, Friedman JE, Grove K, Aagaard KM. Modulations in the offspring gut microbiome are refractory to postnatal synbiotic supplementation among juvenile primates. BMC Microbiol. 2018;18:28.

33. Zhang Z, Lv J, Pan L, Zhang Y. Roles and applications of probiotic Lactobacillus strains. Appl Microbiol Biotechnol. 2018;102:8135-43.

34. Alakomi HL, Skytta E, Saarela M, Mattila-Sandholm T, Latva-Kala K, Helander IM. Lactic acid permeabilizes gram-negative bacteria by disrupting the outer membrane. Appl Environ Microbiol. 2000;66:2001-5.

35. Richards JD, Gong J, de Lange CFM. The gastrointestinal microbiota and its role in monogastric nutrition and health with an emphasis on pigs: current understanding, possible modulations, and new technologies for ecological studies. Can J Anim Sci. 2005:85:421-35.

36. Wang Y, Cao P, Wang L, Zhao Z, Chen Y, Yang Y. Bacterial community diversity associated with different levels of dietary nutrition in the rumen of sheep. Appl Microbiol Biotechnol. 2017;101:3717-28.

37. Li H, Liang T, Chu Q, Xu F, Li Y, Fu L, Zhou B. Effects of several in-feed antibiotic combinations on the abundance and diversity of fecal microbes in weaned pigs. Can J Microbiol. 2017;63:402-10.

38. Ramanan P, Barreto JN, Osmon DR, Tosh PK. Rothia bacteremia: a 10-year experience at Mayo Clinic, Rochester, Minnesota. J Clin Microbiol. 2014;52: $3184-9$.

39. Liu HB, Jia HM, Yu HT, Li N, Wang S, Zeng XF, Zhang XY, Wang YM, Qiao SY, Ding XL. Dietary supplemented antimicrobial peptide microcin J25 improves the growth performance, apparent total tract digestibility, fecal microbiota, and intestinal barrier function of weaned pigs. J Anim Sci. 2017; 95:5064-76.

40. Bui T, Li G, Kim I, Wen K, Twitchell EL, Hualei S, Ramesh AK, Weiss MD, Yang X, Glark-Deener SG, Choy RK, Yuan L. Effects of Racecadotril on weight loss and diarrhea due to human rotavirus in neonatal Gnotobiotic pigs (Sus scrofa domesticus). Comparative medicine. 2017;67:157-64.

41. Chen YJ, Wu H, Wu SD, Lu N, Wang YT, Liu HN, Dong L, Liu TT, Shen XZ Parasutterella, in association with irritable bowel syndrome and intestinal chronic inflammation. J Gastroenterol Hepatol. 2018;33:1844-52.

42. Li N, Huang S, Jiang L, Wang W, Li T, Zuo B, Li Z, Wang J. Differences in the gut microbiota establishment and metabolome characteristics between low- and Normal-birth-weight piglets during early-life. Front Microbiol. 2018;9:1798.

43. He B, Bai Y, Jiang L, Wang W, Li T, Liu P, Tao S, Zhao J, Han D, Wang J. Effects of oat bran on nutrient digestibility, intestinal microbiota, and inflammatory responses in the hindgut of growing pigs. Int J Mol Sci. 2018;19.

\section{Publisher's Note}

Springer Nature remains neutral with regard to jurisdictional claims in published maps and institutional affiliations.

Ready to submit your research? Choose BMC and benefit from:

- fast, convenient online submission

- thorough peer review by experienced researchers in your field

- rapid publication on acceptance

- support for research data, including large and complex data types

- gold Open Access which fosters wider collaboration and increased citations

- maximum visibility for your research: over $100 \mathrm{M}$ website views per year

At $\mathrm{BMC}$, research is always in progress.

Learn more biomedcentral.com/submissions 